Research Article

\title{
Power Efficiency of Energy Harvester Driven by Harmonic Excitation with Amplitude Perturbation
}

\author{
Krzysztof Kucab iD and Grzegorz Górski \\ Faculty of Mathematics and Natural Sciences, University of Rzeszów, Pigonia 1, 35-310 Rzeszów, Poland \\ Correspondence should be addressed to Krzysztof Kucab; kkucab@ur.edu.pl
}

Received 21 December 2018; Revised 22 March 2019; Accepted 8 April 2019; Published 7 May 2019

Academic Editor: Zhonghua Yao

Copyright (C) 2019 Krzysztof Kucab and Grzegorz Górski. This is an open access article distributed under the Creative Commons Attribution License, which permits unrestricted use, distribution, and reproduction in any medium, provided the original work is properly cited.

In this paper, we examine the influence of the background's stochastic excitations on an output power generated by using an energy harvester. The harvester is composed of two magnets attached to a piezoelastic oscillators separated by a distance $\Delta$ from the static magnets fastened directly to the device. We also introduce the parameter $\alpha$ which describes the mass ratio of moving magnets. We examine the output power for different excitation frequencies, different values of $\alpha$, and different amplitudes $\delta_{0}$ of the stochastic force. We also analyze the influence of $\delta_{0}$ and $\Delta$ on the effective output power (EOP), the mean value of output power averaged over the considered frequencies, produced by using the harvester. We have observed that increasing $\delta_{0}$ causes the growth of generated mean power, especially in the low-frequency regime, while the maximum power near the resonance frequency remains unchanged. The EOP also grows with increasing $\delta_{0}$ for all examined values of $\alpha$. The environment's stochastic behavior improves slightly the harvester's efficiency as compared to the purely harmonic case. Analyzing the dependence of EOP on $\Delta$, we observed the maximum which appears at values of $\Delta$ corresponding to the situation when the system starts to work in the unsynchronized regime.

\section{Introduction}

In the world of modern electronic devices which are characterized by very low-power consumption, the energy harvesting devices seem to be very important and useful energy sources, especially in vibrating, hard to reach environments [1-4]. As is well known, the power generated by using an energy harvester in a simple, idealized harmonic case is relatively high only at the resonance frequency. This phenomenon hinders the wider use of such systems. One should take into account that an energy harvesting devices should be adapted to environments which are characterized by a broad frequency band. In this case, the harvesters should also relatively produce large power [5]. Many attempts to obtain energy harvesters with an extended frequency bandwidth have been carried out. To extend the frequency range where the generated effective output power is relatively high, one should use the nonlinear systems. Mann and Sims [6] have shown that the use of magnetic levitation in energy harvesters allows for the nonlinear system to extend the frequency bandwidth.

One of the frequently used energy harvester models, exhibiting the nonlinear properties, is the cantilever ferromagnetic beam (beam with magnetic tip mass) which interacts with the magnetic field generated by using the permanent magnets. The piezoelastic device with attached magnets working in the nonlinear regime is very interesting from the point of view of its frequency band. This type of device exhibits the possibility of generating broadband solutions. One can use two types of arrangements of magnets in these devices. In the first case, one uses the magnetic attraction between harvester's beam and two permanent magnets located symmetrically with respect to the beam's equilibrium position [7-12]. In the second case, one uses the piezoelectric cantilever with magnetic tip mass and the repulsion permanent magnet mounted in the beam's equilibrium position $[5,10,13-17]$. 
The energy harvesting device can be adapted to the vibrating system which in real life vibrates in some spectrum of frequencies and additionally, the vibrations have the stochastic origin $[7,9,11,13,14,18,19]$. Vocca et al. [13] introduced the stochastic term in the equation of motion of the moving magnet. The noise they considered was obtained in an experimental way. In turn, Martens et al. [9] studied the efficiency of vibrating energy harvesting systems with stochastic ambient excitations by solving corresponding Fokker-Planck equations. The stochastic, nonlinear oscillators were also considered in [14, 20-22].

One of the methods used to obtain more efficient power generation in the energy harvesting devices is to use an array of two or more harvesters [23, 24]. Litak and coauthors [25] considered two piezomagnetoelastic oscillators coupled using an electric circuit and driven by harmonic excitations. They used the attracting magnets. They have shown that higher noise levels will favor higher potential barriers, what leads to higher voltage amplitudes and higher power output. In our previous paper [24], we also considered the system consisting of two cantilever beams repelled by using magnets. The harvesting device was excited to vibrations only by the harmonic excitations.

In turn, Rosselló et al. [26] tried to improve the performance of energy harvesting considering a ring of linearly coupled, monostable nonlinear oscillators acting as an energy harvester-through piezoelectric transduction-of mesoscopic fluctuations, which were modeled as Ornstein-Uhlenbeck noise. The main conclusion of their work is that "when several harmonic springs are linearly and bidirectionally coupled to form a ring, it is found that counterphase coupling can largely improve the performance while in-phase coupling worsens it."

There were also attempts to investigate the nonlinear energy harvesters with asymmetric bistable $[12,27]$ and tristable potential [28]. As one can see in these papers, the asymmetric potential bistable energy harvesters are more likely to achieve high-energy branch with initial conditions in the shallower potential well. Additionally, experiments demonstrate that the output performance will be improved for the asymmetric potential harvesters if the initial oscillations are from the shallower potential well. Similar results are also achieved for the tristable potential. Cao and coauthors stated "experiment results reveal that the geometry parameters of the multi-stable configuration can alter the potential function of tristable energy harvesters. Moreover, the shallower potential well depth will enhance the broadband performance and the capability of harvesting energy from low frequency ambient vibration."

In our recent model, at first, we will take into account the nonlinearity of the system. Additionally, we will analyze the influence of stochastic behavior of the environment in which the energy harvesting system is placed. We will analyze the influence of three parameters on the electric power generated by the device. These parameters are the variable distance $\Delta$ between the moving and stable magnets, the mass disproportion parameter $\alpha$ describing the mass ratio of the moving magnets, and the factor $\delta_{0}$ which transforms the system from the nonstochastic regime to the mixed one, which allows us to consider the system in a more realistic way. This work is based on the model described in our previous papers $[12,24]$, where we have analyzed the electrical power generated by using the energy harvesting device subjected to the harmonic excitations only. In this paper, we extend the previous approach for the stochastic behavior of the background vibrations.

\section{Materials and Methods}

In this paper, we study the system consisting of two small magnets attached to the piezoelastic oscillators which move in the magnetic field produced by using the static magnets fastened to the device. The moving magnets are coupled to each other with an electrical circuit. The whole device is subjected to vibrations, which may have a harmonic or stochastic origin. The similar system of two cantilever beams stimulated only by the harmonic excitation force was considered, e.g., Litak et al. [25] and Kucab et al. $[12,24]$. The schematic diagram of our model is presented in Figure 1.

The moving magnets with masses $m_{1}$ and $m_{2}$, respectively, are attached to the piezoelastic beams and are separated from the static magnets by an air gap of thickness $\Delta$. The distance $\Delta$ is responsible for creating the bistable state of magnets' potential energy. As we have shown in our previous paper [12] in the two cantilever beam systems, the best power efficiency is achieved when the distances between beams and magnets are equal $\left(\Delta_{1}=\Delta_{2} \equiv \Delta\right)$. Such an optimal arrangement of beams and magnets will be used in this paper. The masses vibrate in the quasi-horizontal direction described by the variables $x_{1}$ and $x_{2}$, respectively, which indicate the displacements of moving magnets from their equilibrium positions. The distance between static magnets should be large enough to avoid the mutual influence of the moving magnets and also to avoid the interaction between the moving magnet with the magnetic field of the second static magnet (i.e., the magnet which is not directly under the moving one). The mass disproportion parameter $\alpha$ is defined by the following relation: $\alpha=m_{2} / m_{1}$.

As we mentioned earlier, the kinetic excitation, $\tilde{x}_{0}(t)$, is applied to the harvester (we use the notation, where the tilde sign over the symbol indicates the stochastic behavior of corresponding physical quantity). The voltage generated by the piezoelastic beams is transformed into the energy produced on the load resistance $R$ coupled to the device with an electrical circuit. The beam resistor transducer is characterized by the time constant $\tau=R\left(C_{1}+C_{2}\right)$, where $C_{1}$ and $C_{2}$ are the beams' capacitances, presented in Figure 1 for simplicity as the capacitors. The system's physics is described by the set of differential equations which couple the mass displacements of moving magnets $\left(x_{1}, x_{2}\right)$ and the voltage $V$ generated on the electric load. The dynamics of the tip masses motion is described by using Newton's law: 


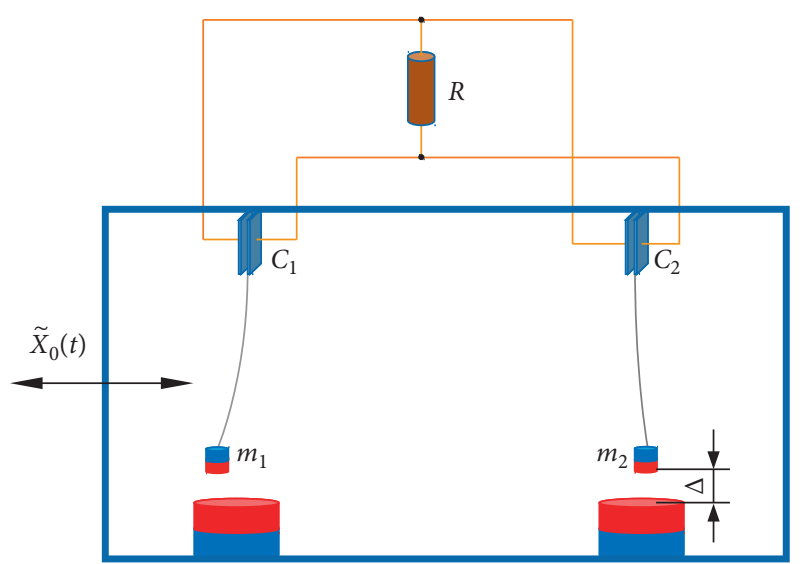

Figure 1: Schematic of the device in the current study.

$$
\left\{\begin{array}{l}
m_{1} \ddot{x}(t)=-\frac{d U(x, \Delta)}{d x}-\gamma \dot{x}-\chi V(t)+\widetilde{F}(t), \\
\alpha m_{1} \ddot{y}(t)=-\frac{d U(y, \Delta)}{d y}-\gamma \dot{y}-\chi V(t)+\widetilde{F}(t) .
\end{array}\right.
$$

The equation for the electrical potential $V(t)$ is given by

$$
\dot{V}(t)=-\frac{1}{\tau} V(t)+\kappa \dot{x}+\kappa \dot{y},
$$

where the overdot sign means the time derivative, $\gamma$ is the viscous parameter, $\chi$ is the piezoelectric coupling constant, and $\kappa$ is the position to voltage coupling coefficient. The excitation force, $\widetilde{F}(t)$, proposed by us, has the following form:

$$
\widetilde{F}(t)=F_{0} \cdot\left[1+\delta_{0} \widetilde{\varepsilon}(t)\right] \sin \omega t,
$$

where $F_{0}$ is the amplitude of the harmonic excitation, $\widetilde{\varepsilon}(t)$ is a set of randomly generated numbers (with a mean value equal to 0 , and a standard deviation equal to 1 ) obeying the Gaussian distribution, and $\omega=2 \pi \nu$, where $\nu$ is the applied frequency. The angular excitation frequency $\omega$ and the amplitude of excitation $F_{0}$ are treated by us, for simplicity, as the parameters.

As we can see in equation (3), the excitation force $\widetilde{F}(t)$ consists of a periodic and stochastic term. Because in the real vibrating systems, there also occur the vibrations of a stochastic nature (e.g. [13]), the excitation force (3) has an additional term which modifies the amplitude of harmonic vibrations. The factor $\delta_{0}$ transforms the system from the nonstochastic regime $\left(\delta_{0}=0\right)$ to the mixed one $\left(\delta_{0}=1\right)$. We limit the value of $\delta_{0}$ to 1 to have the maximum values of the stochastic term in equation (3) of the order of 2, i.e., to have the value of $\left|\delta_{0} \widetilde{\varepsilon}(t)\right|$ factor less than 2 for approximately $95 \%$ of randomly generated values. The stochastic force can be realized, e.g., by considering the periodically moving parts exposed to the random shakes. For example, this can be a situation of a rotating car wheel moving on the uneven road, or the helicopter rotor blades rotating in randomly changing engine conditions.

We are aware of the limitations of this form of stochastic force. Of course the noisy environmental signals have a wide spectrum of frequencies, as opposed to periodic signals. Therefore, using a linear system with a discrete spectrum will leave substantial amounts of energy wasted in the rest of the spectrum of the noisy force. Our model is only the simplification of the real systems, and the obtained results are to serve as a starting point of the more advanced analysis.

The potential energy of moving masses is given by [13]

$$
U(x, \Delta)=\frac{1}{2} k_{\text {eff }} x^{2}+\left(a x^{2}+b \Delta^{2}\right)^{-3 / 2} .
$$

The potential energy $U(y, \Delta)$ has the same form as in equation (4) because we assume that the beams have the same physical properties.

The parameters $k_{\mathrm{eff}}, a$, and $b$ represent the physical properties of piezoelastic beams, and the $\Delta$ parameter (Figure 1) is responsible for transferring the system from the linear regime $(\Delta>15 \mathrm{~mm})$ to the nonlinear (bistable) one.

The potential energy of the oscillators given by using equation (4) is shown in Figure 2. The minimum values of the potential energy, different for each curve, were set to zero (normalized case).

The distance $\Delta$ influences the shape of potential energy $U(x, \Delta)$. For small values of $\Delta$, we obtain two local minima of the potential energy and one local maximum (the potential barrier) at $x=0$. In this case, the system corresponds to a bistable oscillator. For large values of $\Delta$, we have a linear system, corresponding to a monostable oscillator with one local minimum at $x=0$. We can obtain the condition for transition from the bistable system to the linear one calculating the second derivative of potential energy at $x=0$. For $d^{2} U /\left.d x^{2}\right|_{x=0}>0$, we have the bistable potential, and for $d^{2} U /\left.d x^{2}\right|_{x=0}<0$, we have the linear system. At $d^{2} U /\left.d x^{2}\right|_{x=0}=0$, we obtain the critical value of transition from the linear to the bistable system:

$$
\Delta_{\mathrm{cr}} \equiv\left(\frac{3 a}{k_{\mathrm{eff}}}\right)^{1 / 5} b^{-1 / 2} \text {. }
$$

Taking into account the values of parameters presented in Table 1 , we have $\Delta_{\text {cr }} \approx 11.17 \mathrm{~mm}$, at which the potential barrier starts to "appear" at $x=0$.

The most interesting case occurs when the average mechanical energy of the moving magnet is comparable to the value of potential energy barrier at $x=0$. We can estimate the value of $\Delta$ taking into account the stable (nonresonance) case, i.e., when the excitation frequency is low. The calculations show $\left(\delta_{0}=0\right)$ that $x_{\max }=F_{0} / k_{\text {eff }} \approx 6.6 \mathrm{~mm}$, and hence, $U_{\max }=1 / 2 k_{\mathrm{eff}} x_{\max }^{2} \approx 0.58 \mathrm{~mJ}$. This value of energy barrier corresponds to $\Delta_{\text {cr }} \approx 7 \mathrm{~mm}$. As was shown in our previous paper [24], the effective output power has nonzero values for the whole range of considered frequencies for $\Delta=7 \mathrm{~mm}$, while for $\Delta=5 \mathrm{~mm}$, the effective output power is "cutoff" for low-frequency regime.

\section{Results and Discussion}

We perform the computations using the parameters used in [24], where the corresponding nonstochastic system has been examined. These parameters are presented in Table 1. 


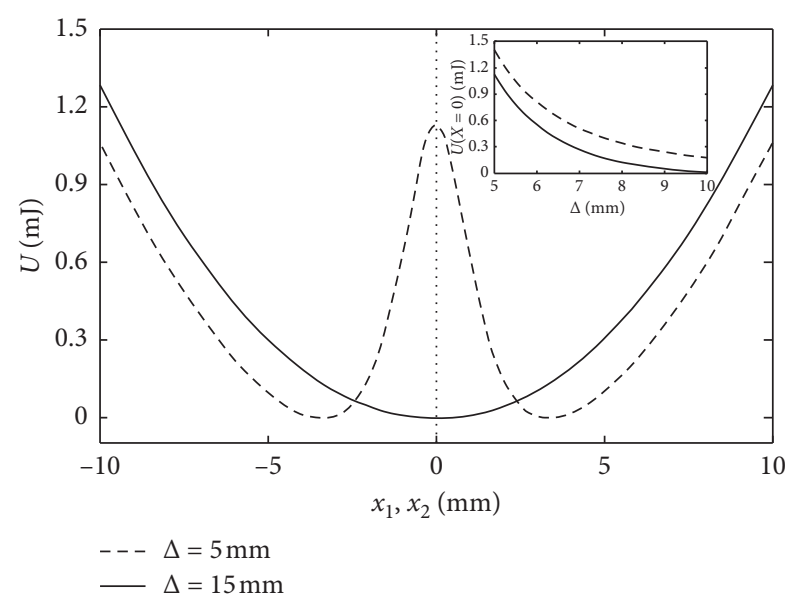

Figure 2: The potential energy $U$ of oscillators versus oscillating mass displacements $x_{1}, x_{2}$ for different values of air gap thickness $\Delta$. Inset: the potential energy versus parameter $\Delta$ at $x=0$ in the normalized case (solid line) and without normalization (dotted line).

TABle 1: The parameters used in our model.

\begin{tabular}{lcc}
\hline Variable name & Variable value & Variable unit \\
\hline$\alpha$ & $1.0 \div 1.2$ & - \\
$\delta_{0}$ & $0.0 \div 1.0$ & - \\
$\Delta$ & $5 ; 15$ & $\mathrm{~mm}$ \\
$\gamma$ & $22 \cdot 10^{-3}$ & $\mathrm{~kg} / \mathrm{s}$ \\
$\chi$ & $1.1 \cdot 10^{-3}$ & $\mathrm{~N} / \mathrm{V}$ \\
$\tau$ & $33.6 \cdot 10^{-3}$ & $\mathrm{~s}$ \\
$R$ & $300 \cdot 10^{3}$ & $\Omega$ \\
$\kappa$ & $4.15 \cdot 10^{3}$ & $\mathrm{~V} / \mathrm{m}$ \\
$m_{1}$ & $18 \cdot 10^{-3}$ & $\mathrm{~kg}$ \\
$k_{\text {eff }}$ & 26.6 & $\mathrm{~N} / \mathrm{m}$ \\
$a$ & $2.82 \cdot 10^{7}$ & $\mathrm{~J}^{-2 / 3} \mathrm{~m}^{-1}$ \\
$b$ & $3.19 \cdot 10^{6}$ & $\mathrm{~J}^{-2 / 3} \mathrm{~m}^{-1}$ \\
$F_{0}$ & $176.6 \cdot 10^{-3}$ & $\mathrm{~N}$ \\
\hline
\end{tabular}

Note: -, dimensionless variable.

We will examine the influence of stochastic excitations on the output power generated by using the considered device at different values of the mistuning parameter $\alpha$. As is shown in our previous paper [24], the decrease in the air gap thickness between moving and stable magnets to values less than $\Delta<5 \mathrm{~mm}$ raises a strong potential barrier which prevents the passage of the magnets through the $x_{1}=0, x_{2}=0$ points (at considered value of applied excitation force) to another side of the potential well. Therefore, the value of maximum power generated by using the device in this regime is much smaller compared to the case, when the magnet can pass the potential barrier. So, in this paper, we use the values of the parameter $\Delta=5 \mathrm{~mm}$ (bistable case) and $\Delta=15 \mathrm{~mm}$ (monostable case).

To describe the influence of the stochastic excitations on the moving magnet motion, we use a set of 100,000 random real numbers obeying a normal distribution function. The set of random numbers used by us, with the mean value equal 0 and a standard deviation equal to 1 , which is presented as the probability density histogram, is shown in Figure 3.

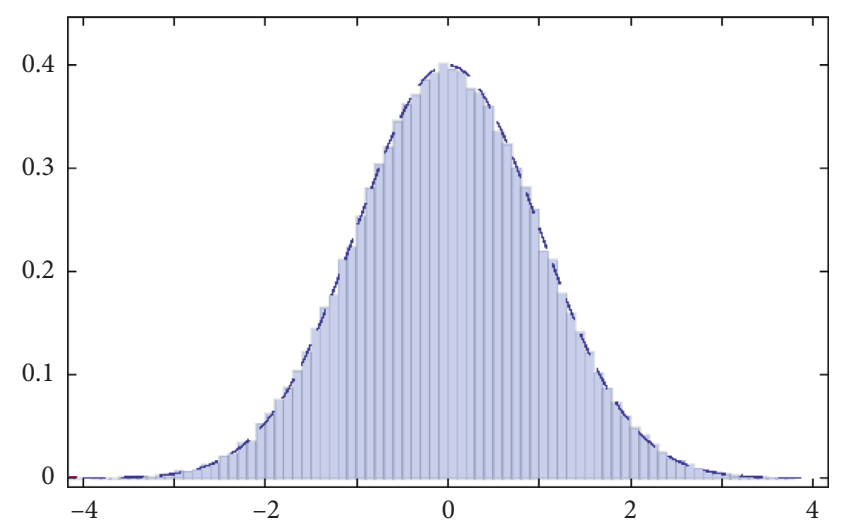

FIgURE 3: The probability density histogram of 100,000 random numbers used in our computations. The dashed line represents a normal distribution function with the mean value equal to 0 and a standard deviation equal to 1 .

In Figure 4, we present the mean electrical power as a function of frequency $v$ for different values of mass disproportion parameter $\alpha$ and for different values of $\delta_{0}$ factor. The mean power is given by the relation $\bar{P}=\left\langle V^{2}\right\rangle / R$, where the $\left\langle V^{2}\right\rangle$ term describes the mean squared voltage generated on the electric load $R$.

As we can see in Figure 4, increasing the value of $\delta_{0}$ factor, i.e., enhancing the stochastic behavior of the external force, causes an increase in the mean output power $\bar{P}$. The increase of $\bar{P}$ with increasing value of $\delta_{0}$ is especially strong in the low-frequency regime where, for harmonic excitations, the output power vanishes. As was mentioned in [24], for low frequencies of harmonic excitation force, the potential barrier (which is the characteristic for small values of $\Delta$ ) stabilizes the pendulum in one equilibrium position. The strong stochastic excitations allow the potential barrier to be overcome by the moving magnet. For the resonance frequency and in the high frequency regime, the contribution from stochastic excitations to the output power is relatively small.

To compare the device's efficiency in the broad frequency range, we show in Figure 5 the effective output power $\langle\bar{P}\rangle$ as a function of the parameter $\delta_{0}$ for different values of $\alpha$. The effective output power is obtained as the average of the mean power over the considered excitation frequencies, $v$, varying from 0 to $15 \mathrm{~Hz}$.

As we can see in Figure 5, the effective output power grows with increasing $\delta_{0}$ for all values of mistuning parameter $\alpha$ considered in this paper. The value of $\alpha>1$ causes that the system has two resonance frequencies which are visible in Figure 4 . The increase of $\alpha$ also slightly broadens the range where the output power is relatively large. On the contrary, the total output power decreases with growing value of the mistuning parameter.

For comparison, in Figure 6, we present the corresponding results at $\Delta=15 \mathrm{~mm}$, i.e., when the device works in the linear regime (monostable case), characterized by the quadratic potential.

The comparison of the effective output power as a function of the parameter $\delta_{0}$ for different values of $\alpha$ for the 


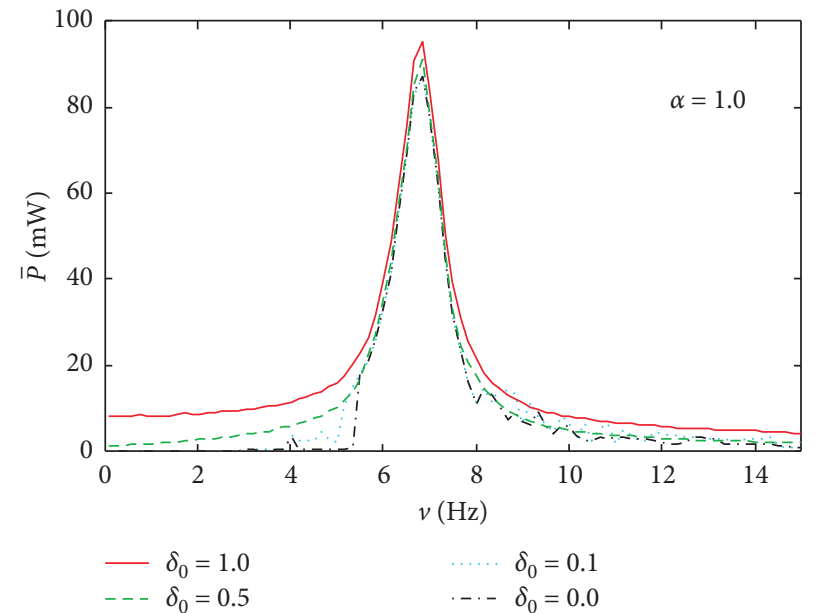

(a)

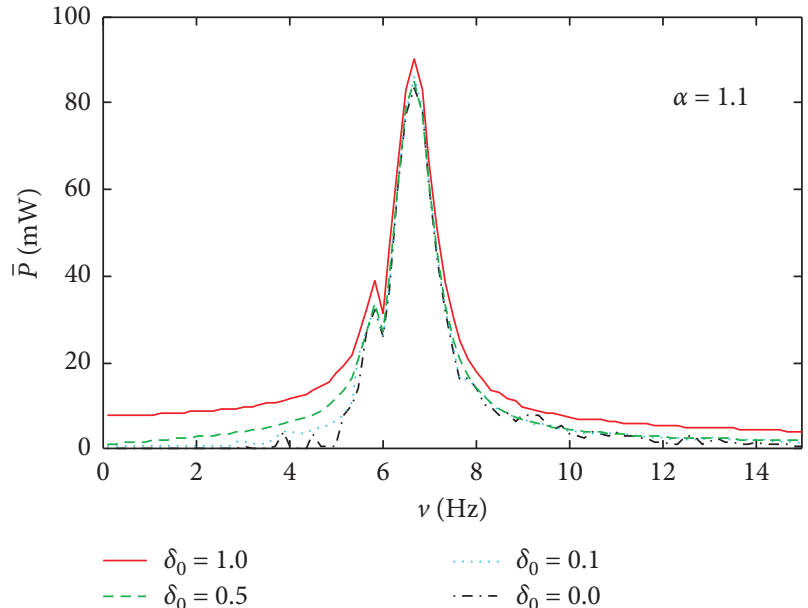

(b)

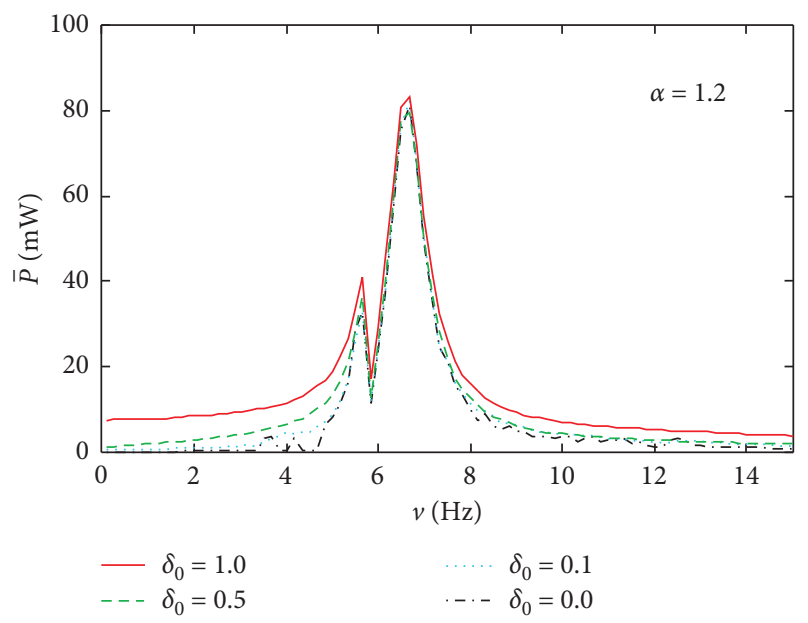

(c)

FIGURE 4: The mean power generated on the electric load $R$ as a function of frequency $v$ for different values of the mass disproportion parameter $\alpha$ and for different values of the stochastic factor $\delta_{0}(\Delta=5 \mathrm{~mm})$.

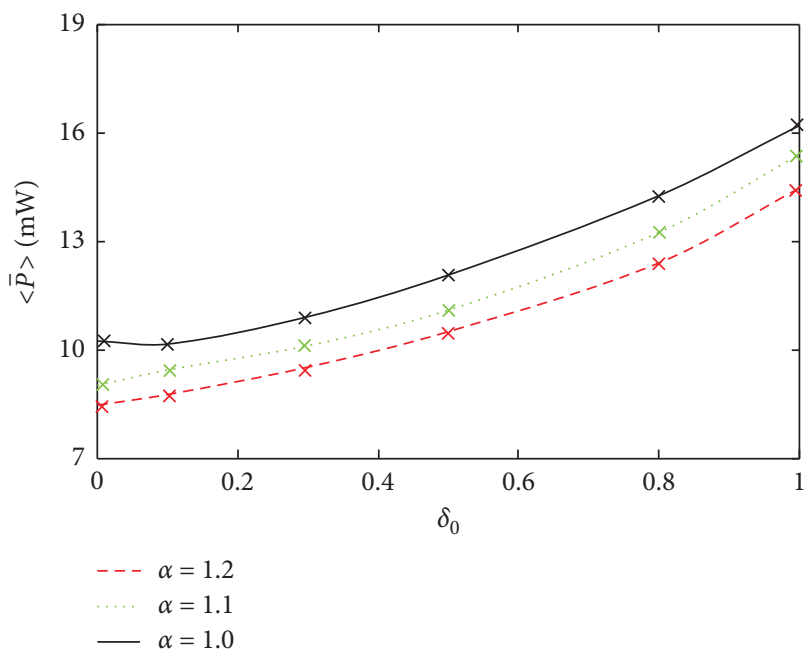

FIgURE 5: The device's effective output power as a function of the parameter $\delta_{0}$ for different values of the mass disproportion parameter $\alpha$ $(\Delta=5 \mathrm{~mm})$. 


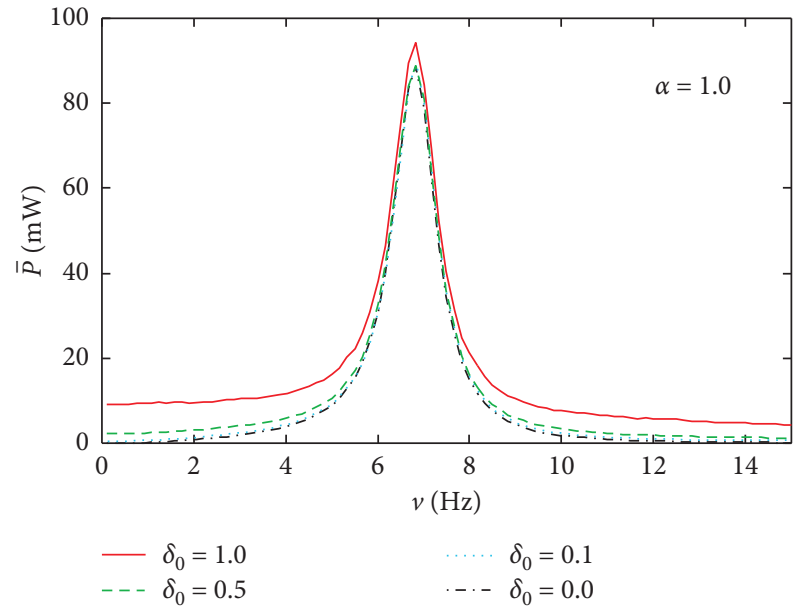

(a)

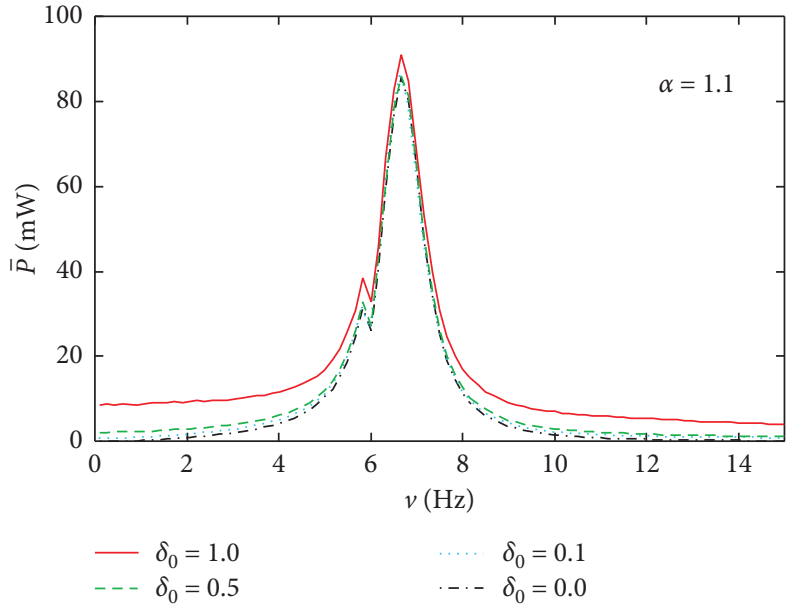

(b)

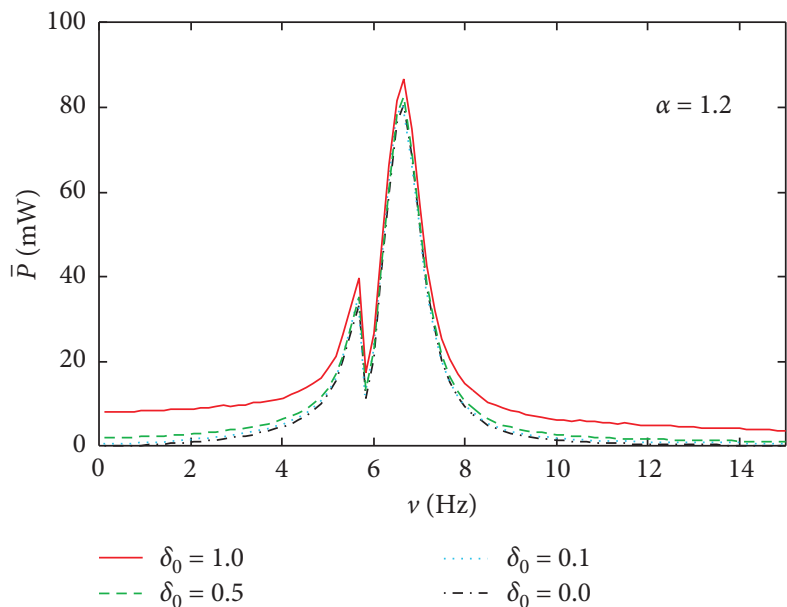

(c)

Figure 6: The mean power generated on the electric load $R$ as a function of excitation frequency $v$ for different values of the mass disproportion parameter $\alpha$ and for different values of $\delta_{0}$ factor $(\Delta=15 \mathrm{~mm})$.

monostable $(\Delta=15 \mathrm{~mm})$ and bistable $(\Delta=5 \mathrm{~mm})$ regimes is presented in Figure 7. As we can see, the results obtained at $\Delta=15 \mathrm{~mm}$ are qualitatively the same as those at $\Delta=5 \mathrm{~mm}$. Introducing the two-minima potential slightly improves the device's efficiency for almost all considered values of the stochastic amplitude $\delta_{0}$.

In Figure 8, we present the influence of the distance $\Delta$ on the effective output power generated on the electric load $R$.

As we can see in Figure 8, for the values of parameter $\Delta<4 \mathrm{~mm}$, i.e., when the moving magnet cannot overcome the potential barrier and it vibrates only on the one side of the static magnet, the effective output power is very small, whereas it grows rapidly with increasing $\Delta$. For $\Delta \sim 5 \mathrm{~mm}$, the value of $\langle\bar{P}\rangle$ reaches maximum and then drops slightly with increasing $\Delta$. The maximum value of the effective output power appears when the kinetic energy of the moving magnet is comparable to the value of the potential barrier at $x=0$. In this case, the system works in the nonlinear regime.

In Figure 9, we present the dependence of the effective output power on the electric load $R$. This dependence helps explaining the effects of coupling between the oscillators on the oscillators harvested power.

As we can see in Figure 9, the optimal value of resistance, for which the generated output power is maximum, is of the order of $R \sim 300 \mathrm{k} \Omega$. The resistance coupling between oscillators causes that the system to behave like the classical power source.

To demonstrate the synchronization of beams, we consider the relative displacements $x-y$ of the oscillators in terms of the standard deviation $\sigma(x-y)$ in function of applied frequency $v$. In Figure 10, we present the dependence of $\sigma(x-y)$ on the applied frequency $v$ for the load resistance equal to $R=300 \mathrm{k} \Omega$. This dependence will explain the effects of coupling between the oscillators on the oscillator dynamics.

As we can see in Figure 10, there are the frequency regions where the oscillators are moving in the opposite direction. For these frequencies, there appears the splitting of the dependence of effective output power versus frequency (Figures 4 and 6) and the effective output power decreases. 


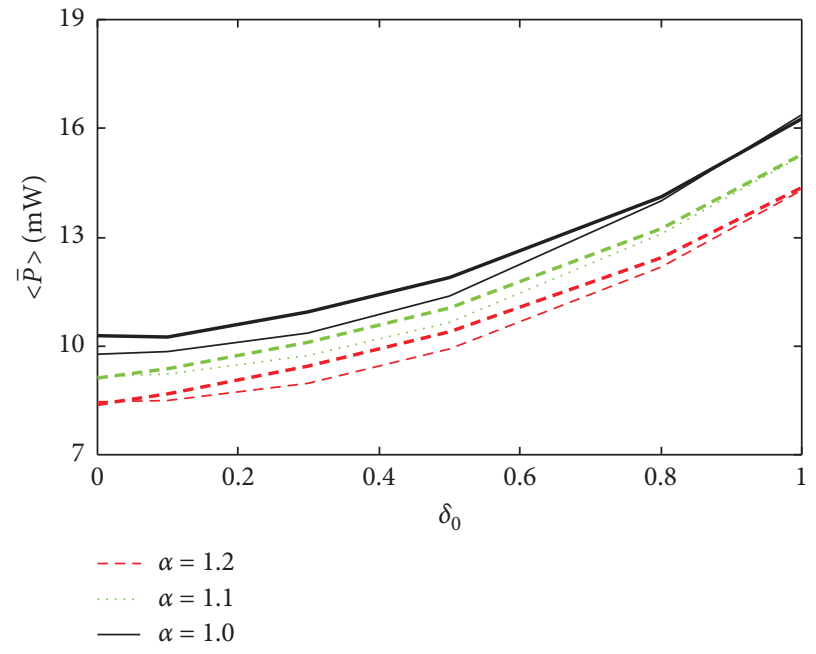

Figure 7: Comparison of the effective output power generated on the electric load $(R)$ as a function of the parameter $\delta_{0}$ for different values of the mass disproportion parameter $\alpha$; thick lines: the bistable case $(\Delta=5 \mathrm{~mm})$; thin lines: the monostable case $(\Delta=15 \mathrm{~mm})$.

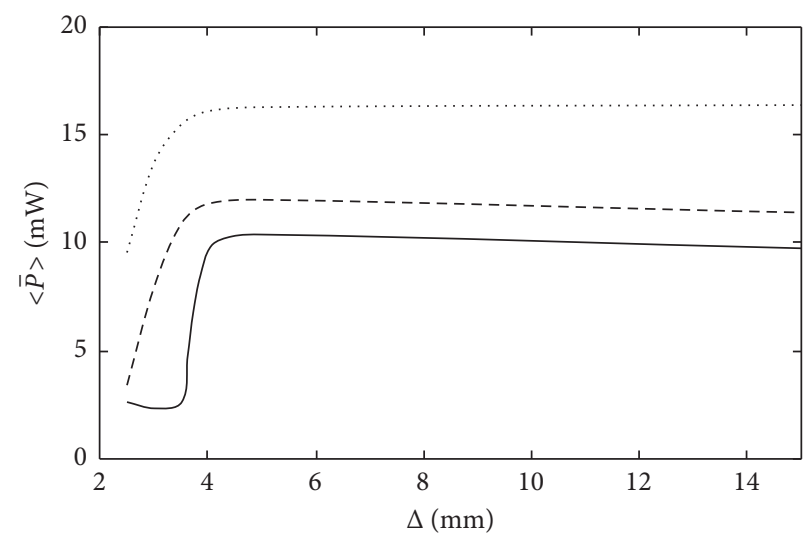

FIgURE 8: The effective output power generated on the electric load $(R)$ as a function of parameter $\Delta$ for $\alpha=1.0$. The solid line is for $\delta_{0}=0.0$, the dashed line is for $\delta_{0}=0.5$, and the dotted line is for $\delta_{0}=1.0$.

It is worth to note here that the relative displacements $x-y$ of the oscillators in terms of standard deviation $\sigma(x-y)$ for $R=30 \Omega$ and $R=3 \mathrm{G} \Omega$ are very similar (almost the same). Due to this fact, we think that the coupling via the load resistance does not influence the oscillators dynamics in a significant way.

To analyze the influence of the load resistance coupling on the effective frequency range, we have considered two types of dependencies. The first one presents the dependence of the frequency range $v_{1 / 2}$ (in $\mathrm{Hz}$ ), for which the effective output power is greater than half of its maximum value, versus the load resistance. The second one presents the dependence of the frequency range $v_{10}$ (in $\mathrm{Hz}$ ), where the effective output power is greater than $10 \mathrm{~mW}$, versus the load resistance. These dependencies are presented in Figure 11.

It is worth to note here that the images in Figure 11(a) are presented on a different scale than the corresponding images in Figure 11(b).
As we can see, the effective frequency range $v_{1 / 2}$ is almost the same for $\delta_{0}=0.0$ and $\delta_{0}=1.0$ in a broad range of considered resistances (Figure 11(a)). It also has similar values for given $R$ for different values of parameters $\alpha$ and $\Delta$. The difference in behavior appears in the dependence of effective frequency range $v_{10}$ (Figure 11(b)). In this case, the values of $v_{10}$ are much greater for $\delta_{0}=1.0$ than that for $\delta_{0}=0.0$ for the values of $R \in(100 \mathrm{k} \Omega ; 1000 \mathrm{k} \Omega)$. We can see that the maximum value of the effective frequency range is achieved for the parameters $\Delta=5 \mathrm{~mm}, \alpha=1.0$. We can also see that the tendency of decreasing of the value of $\nu_{10}$ when $\Delta$ and $\alpha$ increase is opposite for $v_{1 / 2}$. In this case, the effective frequency range increases with the growth of $\alpha$. The effective frequency range is maximum for the value of $R \in(200 \mathrm{k} \Omega ; 400 \mathrm{k} \Omega)$.

All the computations in this work were done with the Mathematica ${ }^{\circledR}$ package. We used the Runge-Kutta algorithm [29]. The simulation time, to reach the self-consistent solution, was chosen to be of the order of one hour when the time interval for sampling the solution was divided into approximately ten million steps. All the average values in numerical integrations were obtained using the Monte Carlo method with the number of steps of the order of one million. The random numbers were generated by using the random number generator included in the Mathematica ${ }^{\circledR}$ package (the same generator was also used in computing the Monte Carlo integrals).

\section{Conclusions}

We have examined the electric power generated by using the energy harvesting device attached to a vibrating source in the case when harmonic excitations are mixed with the stochastic ones. The obtained results were compared to the harmonic case. The bistability of the system was achieved by the relatively small distance between moving and stable magnets $(\Delta=5 \mathrm{~mm})$, so the harvester worked mostly in the nonlinear regime. We have analyzed the total mean power generated on the resistance $R$ as a function of excitation frequency in the harmonic and mixed stochastic regimes. The set of initial conditions corresponding to the stable initial positions of the moving magnets at $t=0$ (i.e., the positions for which the potential energy reaches its minimum) was used.

Increasing the amplitude $\delta_{0}$ of the stochastic force, we have observed the growth of generated mean output power $\bar{P}$, especially in the low-frequency regime. It is interesting that the maximum power near the resonance frequency remained unchanged with the growth of $\delta_{0}$.

The effective output power $\langle\bar{P}\rangle$ was increasing with increasing strength of the stochastic excitation for all examined values of the mistuning parameter $\alpha$, which by itself did not increase the efficiency of the harvester. It was shown that a stochastic behavior of an environment improves slightly the efficiency of the energy harvesting device as compared to a purely harmonic case.

Analyzing the dependence of effective output power on the parameter $\Delta$, we observed the maximum appearing at values of $\Delta$ corresponding to the case when the system starts to work in the unsynchronized regime. 


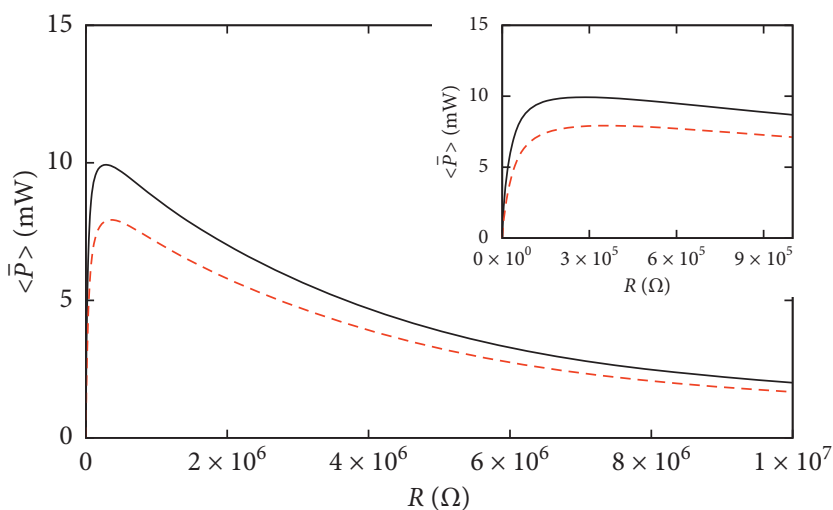

$-\alpha=1.0$

$--\alpha=1.2$

(a)

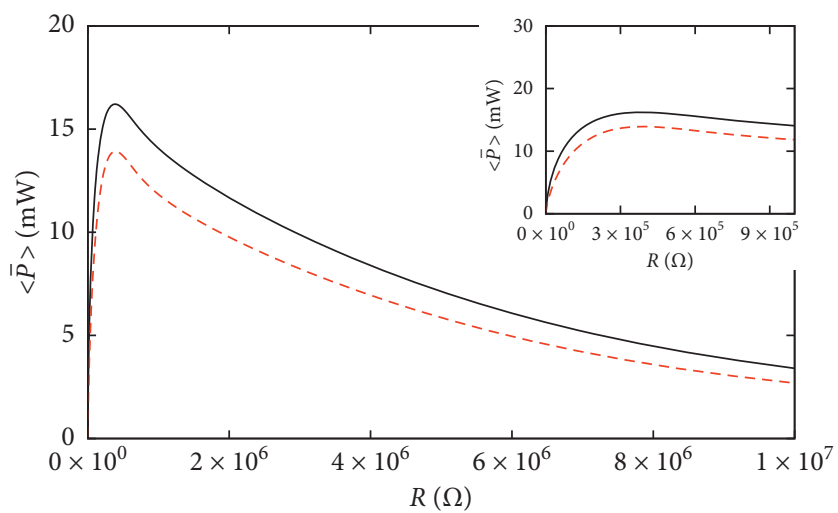

$-\alpha=1.0$

$--\alpha=1.2$

(c)

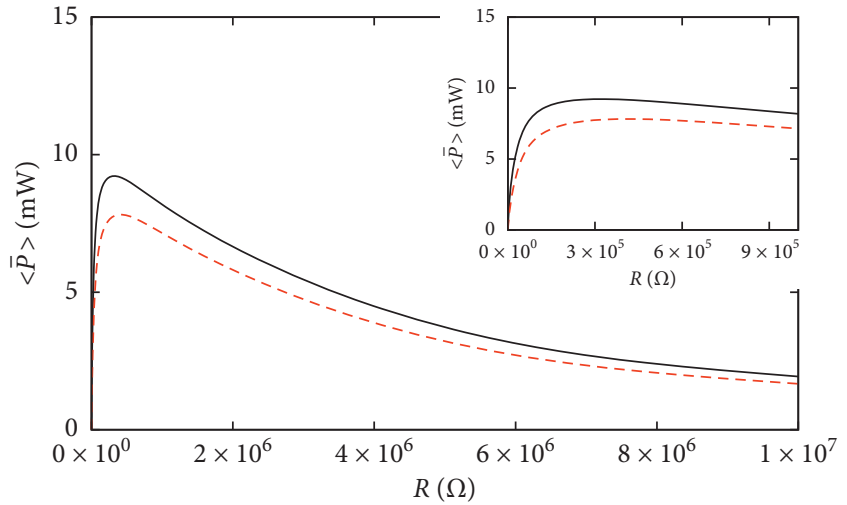

$\alpha=1.0$

$--\alpha=1.2$

(b)

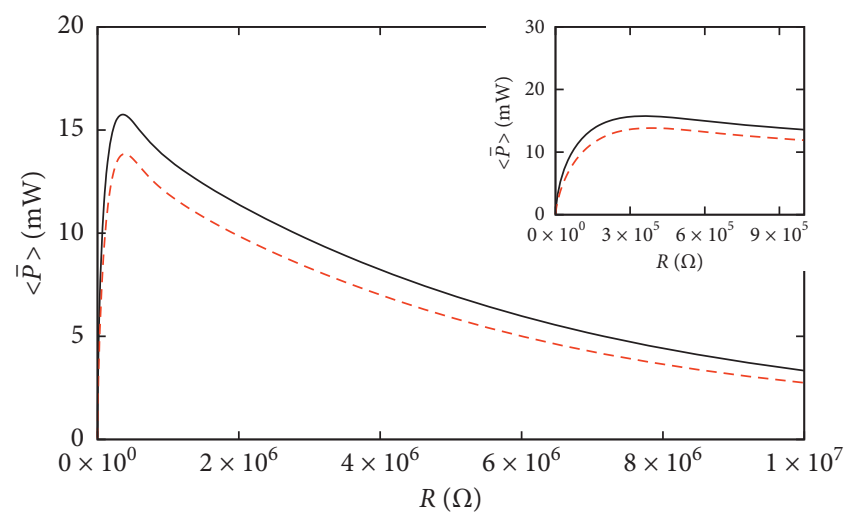

$\alpha=1.0$

(d)

Figure 9: The effective output power generated on the electric load as a function of the load resistance; (a) $\Delta=5 \mathrm{~mm}, \delta_{0}=0.0$; (b) $\Delta=15 \mathrm{~mm}, \delta_{0}=0.0$; (c) $\Delta=5 \mathrm{~mm}, \delta_{0}=1.0 ;$ (d) $\Delta=15 \mathrm{~mm}, \delta_{0}=1.0$.

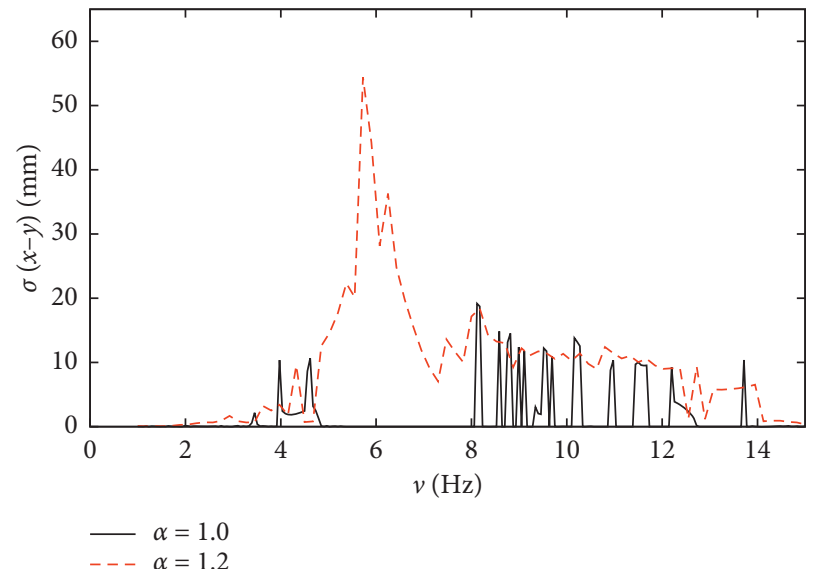

(a)

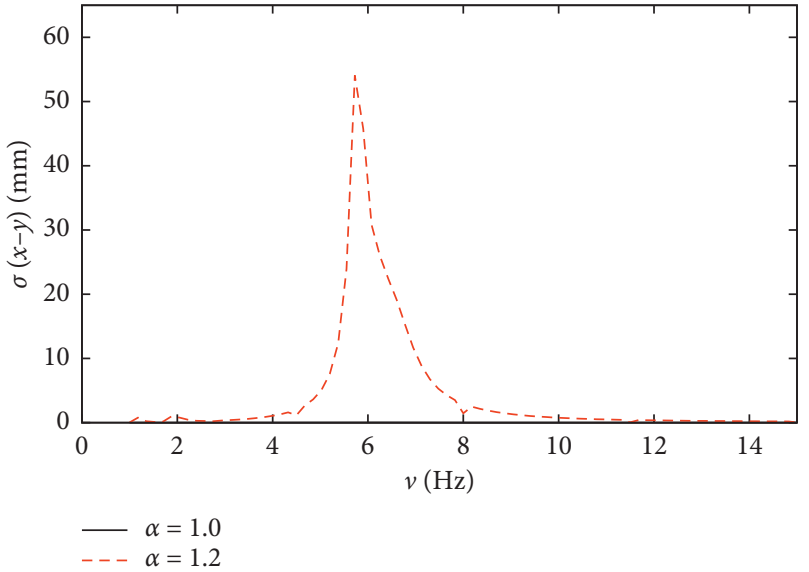

(b)

FIGURE 10: The relative displacements $x-y$ of the oscillators in terms of the standard deviation $\sigma(x-y)$ for $R=300 \mathrm{k} \Omega$ and $\delta_{0}=0.0$ : (a) $\Delta=5 \mathrm{~mm}$; (b) $\Delta=15 \mathrm{~mm}$ (the line for $\alpha=1.0$ is on the $x$-axis). 

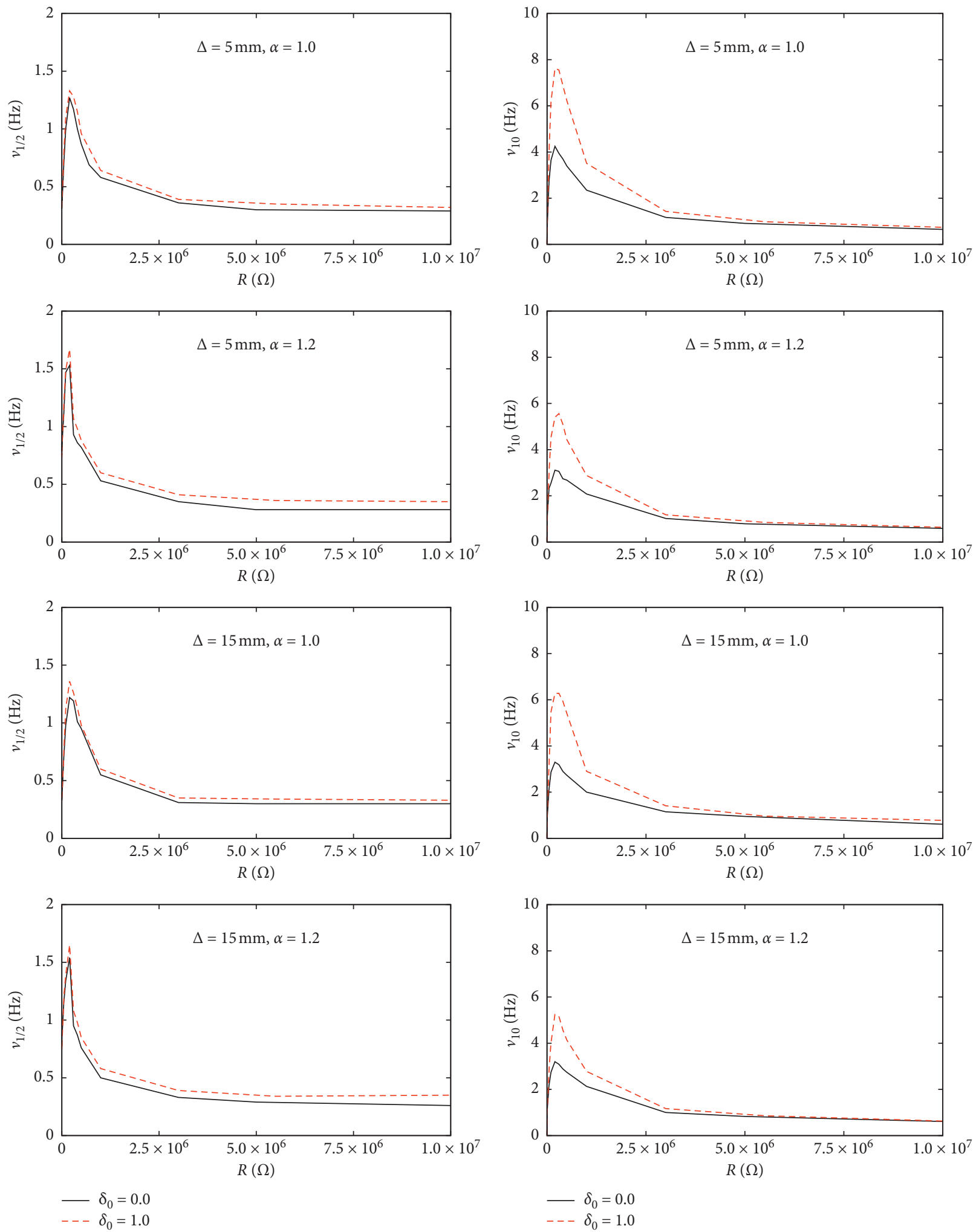

(a)

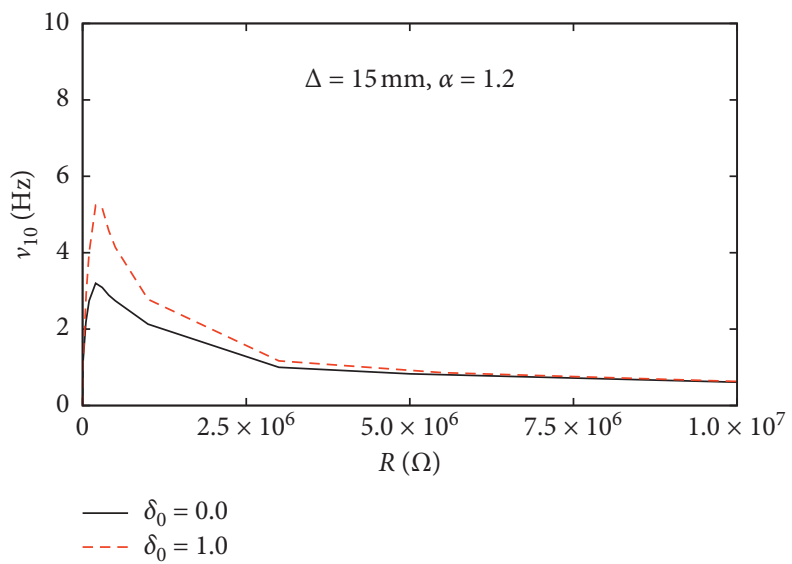

(b)

FIGURE 11: The effective frequency range of the oscillators versus the load resistance $R$.

We also analyzed the dependence of the effective output power on the electric load $R$. We have found that the optimal value of resistance, for which the generated output power is maximum, is of the order of $R \sim 300 \mathrm{k} \Omega$. The resistance coupling between oscillators causes the system to behave like the classical power source. To demonstrate the effects of 
coupling between the oscillators on the oscillators dynamics, we considered the relative displacements $x-y$ of the oscillators in terms of standard deviation $\sigma(x-y)$ in function of applied frequency $\nu$. There are the frequency regions where the oscillators are moving in the opposite direction. In this case, there appears the splitting of the dependence of effective output power versus frequency and the effective output power decreases. Numerical analysis showed that the load resistance does not influence the oscillator dynamics in a significant way.

The computations also showed that the effective frequency range is maximum for the value of $R \in$ $(200 \mathrm{k} \Omega ; 400 \mathrm{k} \Omega)$.

\section{Data Availability}

The data used to support the findings of this study are included within the article.

\section{Disclosure}

Some of the results obtained in this paper were presented by us at the conference "Innovations in Practice" and in focused session "Innovations in Energy Harvesting" as a part of "Panel Discussions Meetings of the Polish Academy of Sciences, Lublin Branch," Lublin, 2019 (Poland).

\section{Conflicts of Interest}

The authors declare that there are no conflicts of interest regarding the publication of this paper.

\section{Acknowledgments}

This work had received support from Faculty of Mathematics and Natural Sciences, Rzeszów University (project no. WMP/GD-14/2018), and partial support from Center for Innovation and Transfer of Natural Sciences and Engineering Knowledge at the Rzeszów University.

\section{References}

[1] S. P. Beeby, M. J. Tudor, and N. M. White, "Energy harvesting vibration sources for microsystems applications," Measurement Science and Technology, vol. 17, no. 12, pp. R175-R195, 2006.

[2] K. V. Selvan and M. S. Mohamed Ali, "Micro-scale energy harvesting devices: review of methodological performances in the last decade," Renewable and Sustainable Energy Reviews, vol. 54, pp. 1035-1047, 2016.

[3] H. A. Sodano, D. J. Inman, and G. Park, "A review of power harvesting from vibration using piezoelectric materials," The Shock and Vibration Digest, vol. 36, no. 3, pp. 197-205, 2004.

[4] H. A. Sodano, D. J. Inman, and G. Park, "Generation and storage of electricity from power harvesting devices," Journal of Intelligent Material Systems and Structures, vol. 16, no. 1, pp. 67-75, 2005.

[5] S. C. Stanton, C. C. McGehee, and B. P. Mann, "Nonlinear dynamics for broadband energy harvesting: investigation of a bistable piezoelectric inertial generator," Physica D: Nonlinear Phenomena, vol. 239, no. 10, pp. 640-653, 2010.
[6] B. P. Mann and N. D. Sims, "Energy harvesting from the nonlinear oscillations of magnetic levitation," Journal of Sound and Vibration, vol. 319, no. 1-2, pp. 515-530, 2009.

[7] G. Litak, M. Borowiec, M. Friswell, and S. Adhikari, "Energy harvesting in a magnetopiezoelastic system driven by random excitations with uniform and gaussian distributions," Journal of Theoretical and Applied Mechanics, vol. 49, p. 757, 2011.

[8] A. Erturk, J. Hoffmann, and D. J. Inman, "A piezomagnetoelastic structure for broadband vibration energy harvesting," Applied Physics Letters, vol. 94, no. 25, p. 254102, 2009.

[9] W. Martens, U. von Wagner, and G. Litak, "Stationary response of nonlinear magneto-piezoelectric energy harvester systems under stochastic excitation," The European Physical Journal Special Topics, vol. 222, no. 7, pp. 1665-1673, 2013.

[10] R. L. Harne and K. W. Wang, "A review of the recent research on vibration energy harvesting via bistable systems," Smart Materials and Structures, vol. 22, no. 2, p. 023001, 2013.

[11] P. Kumar, S. Narayanan, S. Adhikari, and M. I. Friswell, "Fokker-Planck equation analysis of randomly excited nonlinear energy harvester," Journal of Sound and Vibration, vol. 333, no. 7, pp. 2040-2053, 2014.

[12] K. Kucab, W. Paśko, K. Majchrowski, and G. Górski, "Energy harvesting in the nonlinear piezoelastic systems driven by harmonic excitations with asymmetrical shape of the potential energy," International Journal of Structural Stability and Dynamics, vol. 14, no. 08, p. 1440017, 2014.

[13] H. Vocca, I. Neri, F. Travasso, and L. Gammaitoni, "Kinetic energy harvesting with bistable oscillators," Applied Energy, vol. 97, pp. 771-776, 2012.

[14] F. Cottone, H. Vocca, and L. Gammaitoni, "Nonlinear energy harvesting," Physical Review Letters, vol. 102, article 080601, 2009.

[15] W. O. V. Barbosa, A. S. De Paula, M. A. Savi, and D. J. Inman, "Chaos control applied to piezoelectric vibration-based energy harvesting systems," The European Physical Journal Special Topics, vol. 224, no. 14-15, pp. 2787-2801, 2015.

[16] A. D. Paula, D. Inman, and M. Savi, "Energy harvesting in a nonlinear piezomagnetoelastic beam subjected to random excitation," Mechanical Systems and Signal Processing, vol. 5455, pp. 405-416, 2015.

[17] Y. J. Gao, Y. G. Leng, S. B. Fan, and Z. H. Lai, "Performance of bistable piezoelectric cantilever vibration energy harvesters with an elastic support external magnet," Smart Materials and Structures, vol. 23, no. 9, p. 095003, 2014.

[18] W.-A. Jiang and L.-Q. Chen, "Energy harvesting of monostable Duffing oscillator under Gaussian white noise excitation," Mechanics Research Communications, vol. 53, pp. 85-91, 2013.

[19] S. Zhao and A. Erturk, "Electroelastic modeling and experimental validations of piezoelectric energy harvesting from broadband random vibrations of cantilevered bimorphs," Smart Materials and Structures, vol. 22, no. 1, p. 015002, 2013.

[20] D. H. Hawes and R. S. Langley, "Analysis of the power flow in nonlinear oscillators driven by random excitation using the first Wiener kernel," Journal of Sound and Vibration, vol. 412, pp. 256-269, 2018.

[21] W. Wang, J. Cao, C. R. Bowen, D. J. Inman, and J. Lin, "Performance enhancement of nonlinear asymmetric bistable energy harvesting from harmonic, random and human motion excitations," Applied Physics Letters, vol. 112, no. 21, p. 213903, 2018.

[22] Z. Zhou, W. Qin, W. Du, P. Zhu, and Q. Liu, "Improving energy harvesting from random excitation by nonlinear flexible bi-stable energy harvester with a variable potential 
energy function," Mechanical Systems and Signal Processing, vol. 115, pp. 162-172, 2019.

[23] B. Nana and P. Woafo, "Power delivered by an array of Van der Pol oscillators coupled to a resonant cavity," Physica A: Statistical Mechanics and its Applications, vol. 387, no. 13, pp. 3305-3313, 2008.

[24] K. Kucab, G. Górski, and J. Mizia, "Energy harvesting in the nonlinear two-masses piezoelastic system driven by harmonic excitations," The European Physical Journal Special Topics, vol. 222, no. 7, pp. 1607-1616, 2013.

[25] G. Litak, M. I. Friswell, C. A. Kitio Kwuimy, S. Adhikari, and M. Borowiec, "Energy harvesting by two magnetopiezoelastic oscillators with mistuning," Theoretical and Applied Mechanics Letters, vol. 2, no. 4, p. 043009, 2012.

[26] J. I. Peña Rosselló, H. S. Wio, R. R. Deza, and P. Hänggi, "Enhancing energy harvesting by coupling monostable oscillators," The European Physical Journal B, vol. 90, p. 34, 2017.

[27] W. Wang, J. Cao, C. Bowen, and G. Litak, "Multiple solutions of asymmetric potential bistable energy harvesters: numerical simulation and experimental validation," The European Physical Journal B, vol. 91, p. 254, 2018.

[28] J. Cao, S. Zhou, W. Wang, and J. Lin, "Influence of potential well depth on nonlinear tristable energy harvesting," Applied Physics Letters, vol. 106, no. 17, p. 173903, 2015.

[29] A. Naess and V. Moe, "Efficient path integration methods for nonlinear dynamic systems," Probabilistic Engineering $\mathrm{Me}$ chanics, vol. 15, no. 2, pp. 221-231, 2000. 


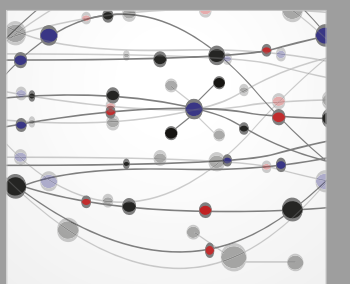

The Scientific World Journal
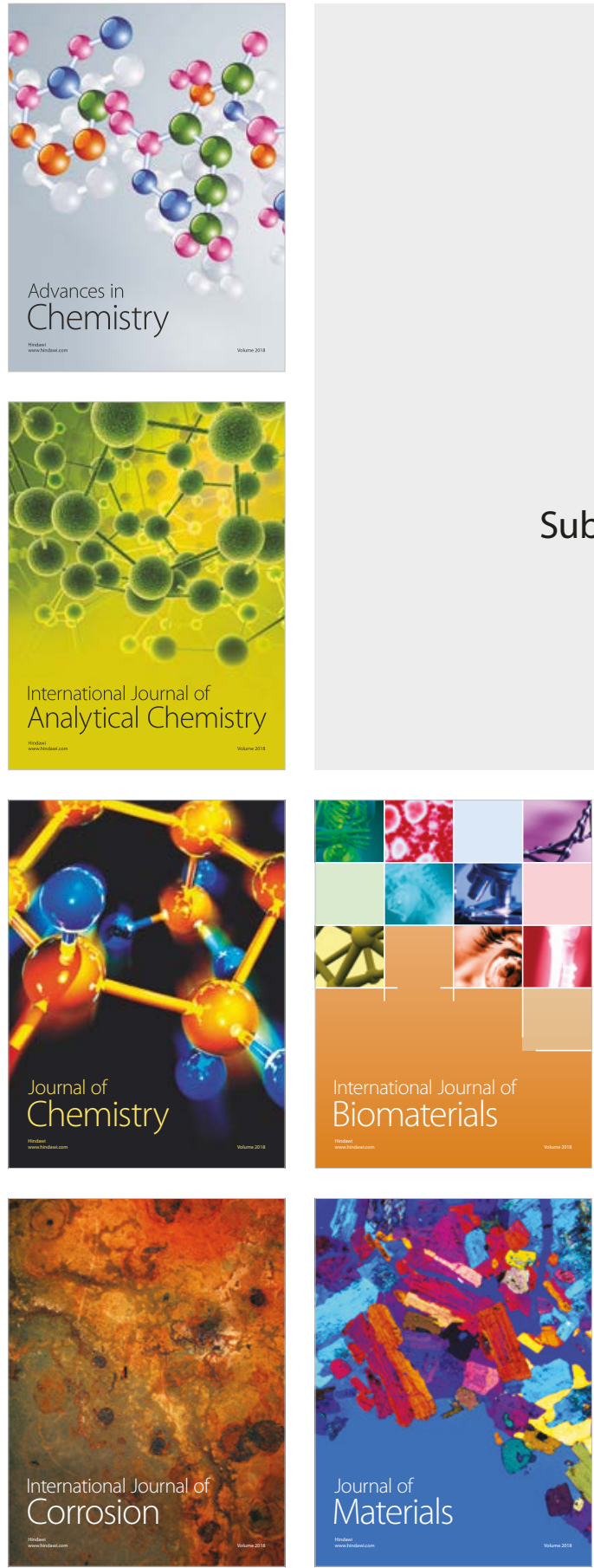

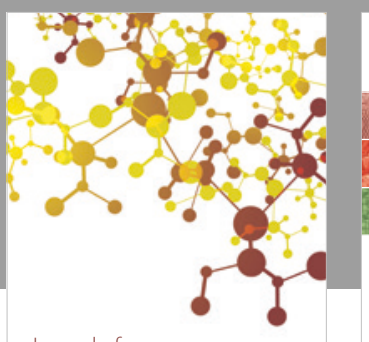

Journal of

Applied Chemistry
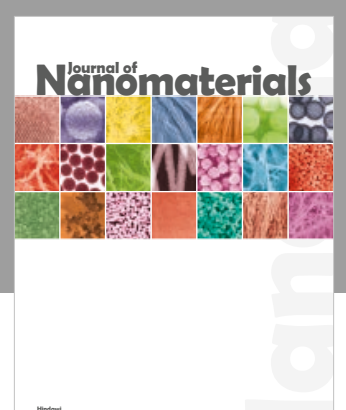

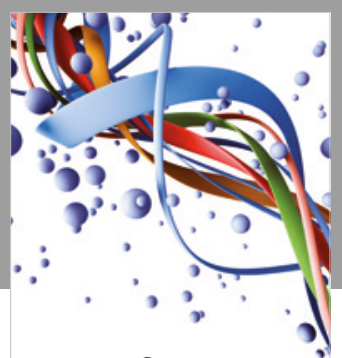

Scientifica

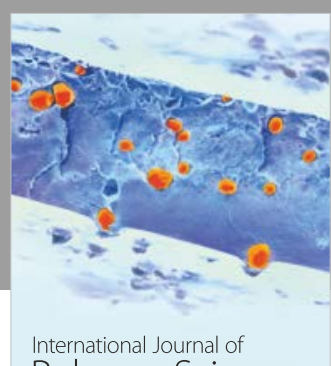

Polymer Science

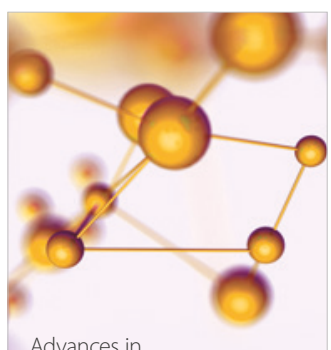

Physical Chemistry
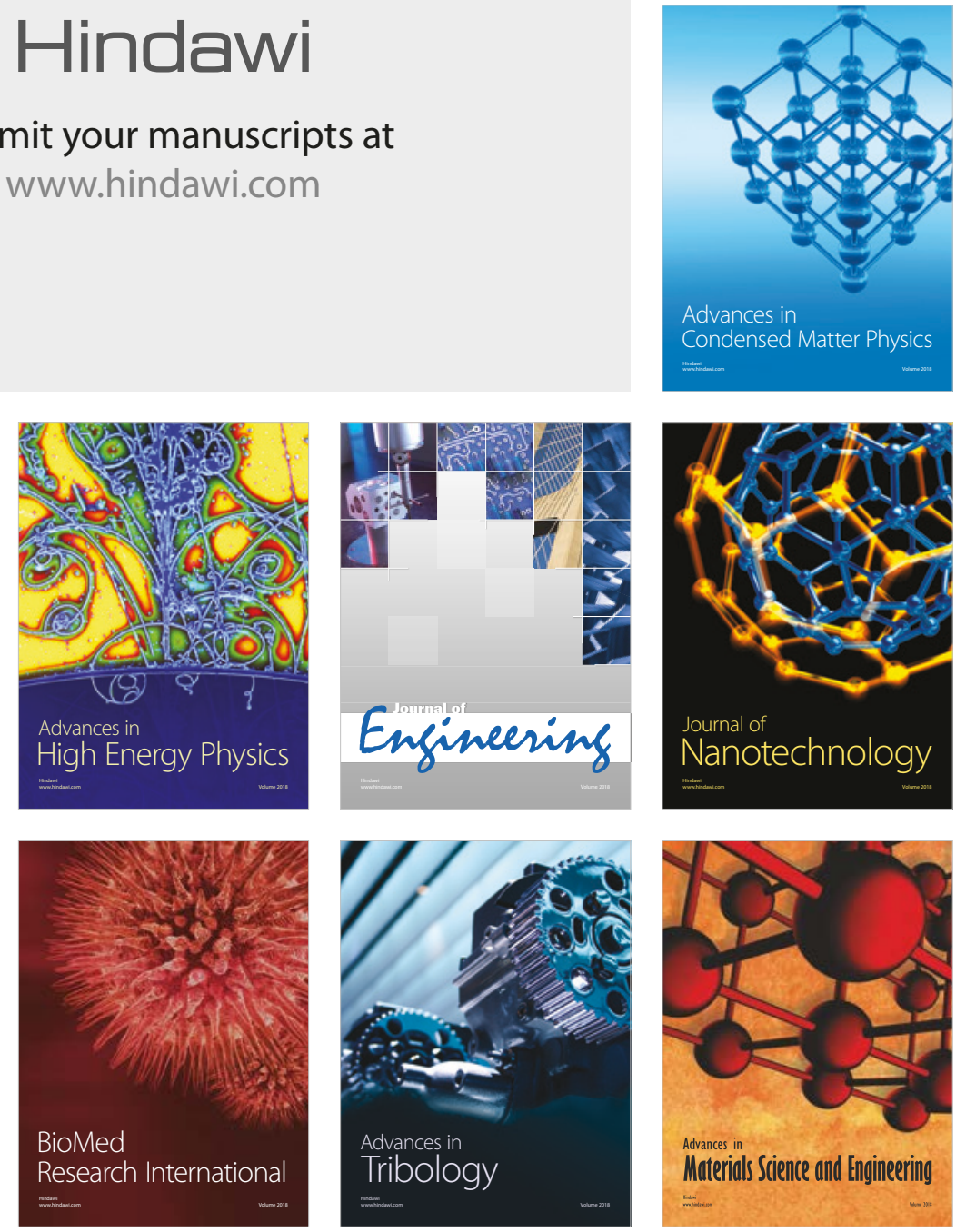